\title{
Sollte man die Angst abschaffen? Warum? Warum nicht? Planung für ein philosophisches Gespräch in einer 5. Klasse
}

\section{Eva Zoller Morf, Beat Baumann (Kreuzlingen, Switzterland)}

An der Pädagogischen Hochschule Thurgau PHTG in Kreuzlingen, approved Schweiz, gibt es das Fach ?Mit Kindern philosophieren? für alle Studierenden der Vor- und der Primarschule schon seit deren Eröffnung im 2003. Zuvor hatte ich als Dozentin die Kinderphilosophie bereits 10 Jahre lang an der Vorläufer-Institution ?Kindergartenseminar Amriswil? unterrichtet, damals gekoppelt mit den Fächern Kinderliteratur und Religion. Zu den Themen einer PwC (Philosophy with Children), wie ich sie an der PH am Bodensee lehre, gehören Fragen aus dem Bereich von Kants Religionsphilosophie (?Was darf ich hoffen??) ebenso, wie solche nach dem Phänomen der menschlichen Gefühle. In den neueren amerikanischen Ansätze von PwC, insbesondere jenem von Thomas Jackson aus Hawaii, wird nicht nur kritisches und kreatives Denken gefördert und gefordert, sondern auch das so genannte ?Caring Thinking?, welches auf Empathie, Fürsorglichkeit, Gerechtigkeitssinn und Gewaltfreiheit fokussiert. Die Studierenden wählen daher für den obligatorischen Praxisversuch des Öfteren auch Fragen rund um Angst oder Mut, Wut oder Glück und manchmal sogar Tod und Trauer, wobei die Gespräche mit den Kindern nicht psychologisch-therapeutisch angelegt sind, sondern zu klarerem Verständnis eines allgemein-menschlichen Phänomens führen sollen.

Hier nun die Planung eines Gesprächs mit Kindern aus einer 5. Klasse, das der Student Beat Baumann in seinem Praktikum 2009 erfolgreich umgesetzt hat. Er folgte bei der Planung meinen Vorgaben, zu denen die Formulierung so genannter ?Hebammenfragen? gehört. Ich nenne die Fragen so in Anlehnung an die mäeutische Methode des Sokrates, wobei aber auf Suggestivfragen zu verzichten ist.

\section{Sollte man die Angst abschaffen? Warum? Warum nicht?}

Praxisplanung von Beat Baumann

\section{Einstiegsvorgehen /-fragen:}

Ich werde den Kindern einer 5. Klasse sagen, dass wir in diesem Gespräch über ein Phänomen nachdenken wollen, das vermutlich alle schon längst kennen. Niemand habe ?es? besonders gern, und so hätte ich mir gedacht, eigentlich könne man es doch abschaffen. Ich erkläre dann auch noch, dass ich daran interessiert sei, die Meinungen der Kinder darüber zu hören.

Zu Beginn werde ich den Kindern von einem eigenen Angsterlebnis erzählen nach dem Schema ?Woher kommt das Gefühl? ? Wie genau ? und unterschiedlich ? kann es sich anfühlen? ? Wohin damit? ? und schliesslich allfällige Bewertungen aller drei Aspekte.? (Anmerkung EZM: Dieses Schema habe ich in meinem Buch ?Philosophische Reise ? Unterwegs mit Kindern auf der Suche nach Lebensfreude und Sinn?, Zürich 2006, beschrieben, und ich lehre es auch so an der PHTG.) Ich werde beschreiben, weshalb ich die Angst verspürt hatte, wie sich dieses Gefühl zeigte, und was ich in diesem Moment am liebsten getan hätte.

Mein Angsterlebnis:

- $\quad$ Alleine auf dem Nach-Hause-Weg im dunkeln Wald.

- Dinge gesehen und gehört im Fernsehen, Radio oder in Büchern.

- $\quad$ Der Gedanke: ?Was wäre, wenn ??? löste in mir die Angst aus.

- Es folgten umherschweifende Blicke, hoher Puls, schneller Schritt, Selbstgespräche, Respekt ?

- und schliesslich die Überlegungen:

? Darf Angst eigentlich sein? Ist man ein ?Angsthase?, wenn man sie zeigt?

Einstieg ins eigentliche Gespräch durch Aktivierung eigener Erfahrungen:

Die Kinder sollen zurückdenken an ihr grösstes oder aktuellstes Angsterlebnis. Sie machen sich in Einzelarbeit Notizen zu den drei Punkten:

- Hergang (Weshalb kam es zum Gefühl?)

- das Gefühl (Wie und wo spürte ich es im Körper?)

- Verlauf des Gefühls (Wie hat es sich gezeigt?)

Im Anschluss an die Einzelarbeit können die Kinder auf freiwilliger Basis in der Runde von ihrem Angsterlebnis erzählen. 


\section{Allgemeine Überlegung:}

Ich fasse von Zeit zu Zeit die Diskussion zusammen und frage nach.

Wichtig ist, dass ich sparsam mit meinen Beiträgen umgehe und den Kindern genügend Raum für ihre eigenen Überlegungen lasse.

Klärung des zentralen Begriffs:

Nachdem die Kinder einige Beispiele erzählt haben, folgen differenzierende Hebammenfragen:

- War die Angst wohl wirklich bei allen genau gleich? Was könnte alles verschieden sein bei euren Angsterlebnissen?

- Z.B. wodurch die Angst entstanden ist, was sie ausgelöst hat?

- Oder wie sie sich angefühlt hat? (War sie ganz stark, oder warst du vielleicht nur ein bisschen ängstlich? Panik? Schreck? ? differenzierte Begriffsarbeit!)

- Vielleicht auch, wie man mit der Angst umgehen könnte? (Was tatet ihr, als ihr die Angst spürtet? Wie unterschiedlich können unsere Reaktionen auf Angst sein?)

- Gibt es eigentlich verschiedene Ängste? Welche? Was ist auch noch so ähnlich? (Furcht, Panik, Schrecken, Gruseln, etc.) Wie unterscheiden sie sich?

- Gibt es Gemeinsamkeiten zwischen diesen verschiedenen Arten von Angst?

- Wie könnten wir ?die Angst? also definieren? Gibt es überhaupt eine Definition? Warum (nicht)?

Ich werde die wichtigsten der genannten Angst-Arten zusammenfassen und erklären:

Wenn wir jetzt dann über das Abschaffen der Angst sprechen werden, müssen wir uns jeweils darüber im Klaren sein, von welcher Angst wir gerade sprechen. Die einen Ängste kann man ja vielleicht wirklich versuchen loszulassen, andere aber sind möglicherweise ganz wichtig für uns. Daraus ergibt sich für uns nun der zweite Teil des Gesprächs.

Wie denken wir über den Wert, den Sinn von Angst?

Sollte man alle Arten von Angst ausrotten? Warum vielleicht (nicht)?

Hebammenfragen zu diesem Gesprächsteil:

? Leben mit Angst:

- Warum hat man eigentlich Angst? Überlegt mal, wie das bei eurem Beispiel war.

- Was sollte die Angst in diesem Beispiel vielleicht bewirken?

- Wann und wie ist die Angst verschwunden? Einfach so? Wirklich?

? Nachteile der Angst:

- Wem stand die Angst schon mal im Weg? Erzählt, wo und wie ihr das erlebt habt? (?Mehrere Beispiele untersuchen)

- Das war sicher nicht sehr angenehm. Aber war die Angst bei deinem Beispiel überflüssig? Weshalb (nicht)? Und bei dir?? Bei dir??

- Könnte man sie nicht einfach abschaffen?

- Wieso glaubt ihr, dass man sie (nicht) abschaffen kann? (?möglichst viele Argumente dafür und dagegen sammeln!)

? Vorteile der Angst:

- $\quad$ Gibt es auch Gutes, das die Angst mit sich bringt?

- Welches denn zum Beispiel? Warum soll das gut sein?

- Hat euch die Angst vielleicht schon mal geholfen?

- Wo und wie denn?

? Gedankenspiel: Leben ohne Angst:

- Was wäre geschehen, wenn du bei der beschriebenen Situation keine Angst verspürt hättest?

- Was wären die Nachteile/Vorteile, wenn man keine Angst mehr hätte?

- Wie stellt ihr euch ein Leben ganz ohne Angst vor?

- Was wäre anders auf der Welt ohne Angst?

- Würde euch das gefallen? Weshalb (nicht)?

- Wäre ein Leben ohne Angst überhaupt denkbar? Wie? Warum nicht? 


\section{Abschluss des Gespräches}

Zusammenfassung des Gespräches durch mich. Auch ein paar Evaluationsfragen, welche die Kinder mit der Daumensprache beantworten, werde ich noch stellen:

- Hast du während dem Gespräch heute etwas Neues gedacht?

- Hast du dich wohl gefühlt und immer getraut, deine Meinung beizusteuern?

- War es unangenehm über die Angst zu sprechen?

- Fandest du das Gespräch interessant?

Danach werde ich den Kindern noch zwei Zitate auf den Weg geben, welche sie zum Weiterdenken anregen sollen:

Den größten Fehler, den man im Leben machen kann, ist, immer Angst zu haben, einen Fehler zu machen.

Dietrich Bonhoeffer

Nur eines macht sein Traumziel unerreichbar: die Angst vor dem Versagen.

Paulo Coelho 